\title{
Nafion-Based Low-Hydration Polyelectrolyte Multilayer Membranes for Enhanced Water Purification
}

\author{
Dennis M. Reurink, Esra te Brinke, Iske Achterhuis, Hendrik D. W. Roesink, and Wiebe M. de Vos*(i) \\ Membrane Science \& Technology, University of Twente, MESA+ Institute for Nanotechnology, P. O. Box 217, 7500 AE Enschede, \\ The Netherlands
}

\section{Supporting Information}

\begin{abstract}
The increase of micropollutant concentration in both surface and groundwater is an emerging concern for the environment and human health. Most of such small organic molecules (medicines, hormones, and plasticizers) enter the environment via our wastewater, because they are not sufficiently removed by the current techniques applied in wastewater treatment plants. A possible solution to remove micropollutants is the usage of polyelectrolyte multilayer (PEM) based membranes. PEM membranes have received a growing interest in the past decade due to their high chemical and physical stability and their high permeability and selectivity. A popular polyelectrolyte pair to make dense PEM membranes with high salt retentions is the combination of poly(allylamine

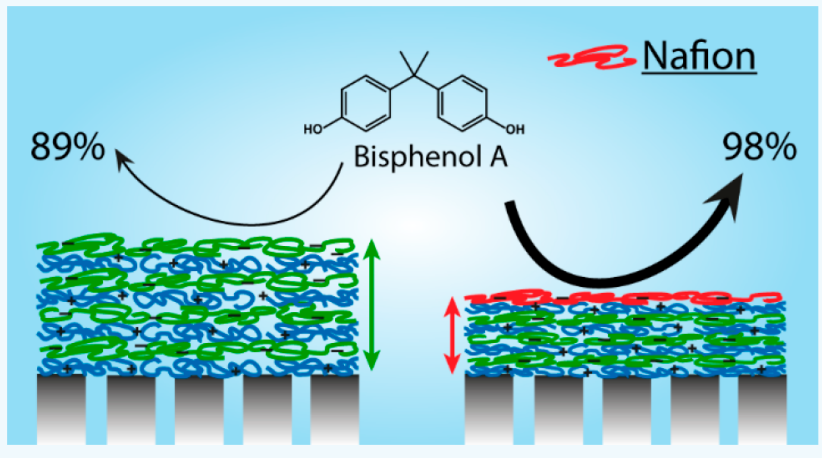
hydrochloride) (PAH) and poly(sodium 4-styrenesulfonate) (PSS). Unfortunately, smaller micropollutants (such as bisphenol A, sulfamethoxazole, naproxen, and bezafibrate) still show significant permeation through this membrane. In this study, for the first time, a single final layer of Nafion is applied on the PEM to increase the density of the PEM membrane. It is shown that when terminating with Nafion, the swelling of the multilayer decreases by $50 \%$. These pronounced changes in layer structure are reflected by changes in membrane performance, such as a lower molecular weight cutoff (MWCO) and an increasing hydraulic membrane resistance. Furthermore, we show that the Nafion content of the multilayer can be increased by constructing a Nafion/PAH multilayer on top of the existing PSS/PAH multilayer, thereby lowering the MWCO. Although hydraulic resistance increases, these PSS/PAH/Nafion-based multilayers show excellent performance in rejecting difficult-to-remove micropollutants that have low molecular weight (200-650 Da) and different charges. Overall, a cocktail of eight small micropollutants can be removed up to $97 \%$ by these membranes, allowing strongly enhanced water purification.
\end{abstract}

KEYWORDS: polyelectrolyte multilayers, nanofiltration, Nafion, swelling, micropollutants

\section{INTRODUCTION}

Growing concentrations of micropollutants in our surface and our drinking water sources lead to increased worries about their long- and short-term effects on human health and the environment. ${ }^{1}$ Waste water from hospitals, industry, and domestic households contains a large variation of small (100-1000 Da) and difficult-to-remove micropollutants (MPs). Unfortunately, traditional wastewater treatment based on flotation, clarification, and (an)aerobic treatment only removes a small amount of these micropollutants from waste streams. $^{2}$ The purified water, still containing MPs, is discharged in rivers and other drinking water resources., To prevent any micropollutants from ending up in our drinking water, membrane filtration of ground and surface water can be a solution to provide clean and safe drinking water. Alternatively, membranes could be used in wastewater treatment to prevent micropollutants from being released into the environment at all. For both possible treatment options, it is clear that better membranes need to be developed in order to remove micropollutants in an effective and efficient way.
In membrane science, various types of filtration exist, namely, microfiltration, ultrafiltration (UF), nanofiltration $(\mathrm{NF})$, and reverse osmosis (RO). Where microfiltration and ultrafiltration are based on pore-flow membranes, reverse osmosis membranes have a dense separation layer and therefore, the solution-diffusion principle applies. ${ }^{5} \mathrm{NF}$ membranes have properties in between RO and UF, but typically share the dense polymeric top layer of RO. NF has been found to separate on principles of Donnan, dielectric, and size exclusion. ${ }^{6,7}$ Nowadays, most NF membranes are thin film composite membranes consisting of a porous support, coated with a dense polyamide separation layer prepared by means of interfacial polymerization (IP). ${ }^{8}$ While these membranes are very successfully applied for, e.g., hardness removal, their preparation technique has limitations. For example, it is very difficult to utilize IP coating for the inside of a hollow fiber

Received: July 25, 2019

Accepted: August 19, 2019

Published: August 19, 2019 


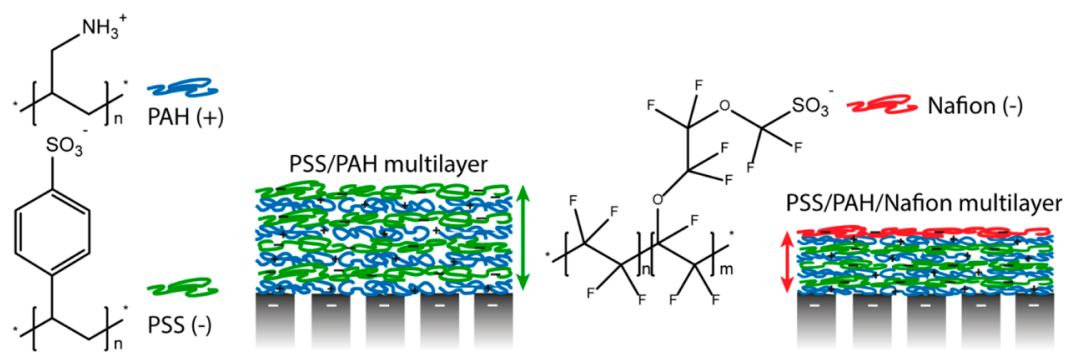

Figure 1. Schematic overview of the studied systems. Here, a PSS/PAH multilayer is constructed from the polyanion PSS and the polycation PAH. Nafion, a very hydrophobic polyelectrolyte, is added as a final layer to reduce the swelling of the multilayer. For a denser multilayer, more selective separation properties are expected.

membrane, while this geometry would hold many benefits in wastewater treatment, such as a high packing density and lower fouling potential. Moreover, IP-based coatings are chemically weak and prone to degrade when treated with chemical cleaning agents. ${ }^{\text {? }}$

An upcoming technique to prepare NF membranes is layerby-layer (LbL) assembly of oppositely charged polyelectrolytes. This highly effective method makes use of alternating deposition of two oppositely charged polyelectrolytes on a support membrane, independent of the support membrane geometry. This allows efficient fabrication of hollow fiber NF membranes, with the separating layer on the inside of the hollow fiber membrane. Furthermore, the LbL technique allows easy fine-tuning of the resulting membrane properties, by control over the used polyelectrolytes ${ }^{10}$ and the coating conditions, such as salt concentration ${ }^{11}$ and $\mathrm{pH}^{12}$ Fabrication of PEM-based hollow fiber membranes can be performed using simple immersive techniques such as dip coating ${ }^{11}$ or dynamic coating $^{13}$ of polyelectrolytes on the surface by flushing the membrane with appropriate polyelectrolyte solutions. To coat a PEM, various charged support surfaces can be used, including sulfonated poly(ether sulfone $)^{9}$ and plasma treated poly(acrylonitrile). ${ }^{14}$ It has been shown that a higher charge density of the support layer creates more stable PEM membranes.'

One commonly used polyelectrolyte pair to manufacture NF membranes is the combination of poly(styrenesulfonate) (PSS) and poly(diallyl dimethylammonium chloride) (PDADMAC). ${ }^{15}$ This system has many advantages such as good permeability, high multivalent ion retention, and a very high physical and chemical stability. However, major drawbacks of PSS/PDADMAC-based PEM membranes are the relatively high molecular weight cutoff (MWCO) and thus low rejections of micropollutants. ${ }^{11,15}$ To obtain better retention performance toward MPs, membranes need to be designed with much more effective size-based exclusion, and thus a lower MWCO. A polyelectrolyte couple that has a denser structure, a high retention toward solutes, and still a high chemical and physical stability is the combination of PSS and poly(allylamine hydrochloric acid) ( $\mathrm{PAH}$ ). This polyelectrolyte couple leads to PEMs with a bulk excess of positive charge, resulting in a high retention toward ions. ${ }^{16,17}$ Furthermore, PSS/PAH-based membranes have a small effective pore size (estimated to be $0.34-0.5 \mathrm{~nm}$, depending on the $\mathrm{pH}$ of $\mathrm{PAH}$ during coating), which results in a high retention toward uncharged solutes. ${ }^{18,19}$ Still, we believe that it is required to densify this layer even further, to attain the required selectivity to remove small and uncharged MPs, such as bisphenol A, sufficiently.
Nafion is a well-known material used for the fabrication of high-performance ion exchange membranes (IEMs) for electrodialysis/electrolysis and fuel cell applications. ${ }^{20,21}$ Indeed, Nafion-based membranes are among the densest ion exchange membranes in production, caused by the hydrophobic nature of the fluorinated polymer, leading to very high ionic selectivities. Even so, the selectivity between monovalent and divalent ions can still be increased by simply coating the surface of IEMs ${ }^{22}$ and more specifically Nafion-based IEMs ${ }^{23}$ with PEM-based coatings. In this study, however, we propose to reverse this approach by utilizing Nafion to achieve denser and more selective PEM-based membranes. It has been shown, on model surfaces, that in combination with PDADMAC or PAH a PEM can be constructed with Nafion acting as the polyanion. $^{24-27}$ Constructed PEMs with Nafion have been shown to be very hydrophobic even when a "normal" PEM is terminated by just a single layer of Nafion, a so-called Janus film. ${ }^{28}$

In this work, we demonstrate for the first time that a Nafion coating can be applied as a post-treatment to create lowhydrated PEM membranes with an increased micropollutant retention. We study how Nafion impacts the swelling and hydrophilicity of a PSS/PAH multilayer and the effects on membrane performance, as shown schematically in Figure 1. To characterize the multilayer, ellipsometry is used to study the swelling of the multilayer before and after terminating with Nafion. In addition, contact angle measurements are performed to study the hydrophilicity. Membrane performance is studied by measuring the hydraulic resistance after each coating step of PSS, PAH, and Nafion. Finally, we will show that through terminating with Nafion, the multilayer has a lower MWCO and a higher retention for micropollutants but will keep the same salt retention behavior.

\section{EXPERIMENTAL SECTION}

Materials. Poly(allylamine hydrochloric acid) (PAH, MW = $150,000 \mathrm{~g} \cdot \mathrm{mol}^{-1}, 40 \mathrm{wt} \%$ in water) was purchased from Nittobo Medical, Japan, while poly(sodium 4-styrenesulfonate) (PSS, MW = $200,000 \mathrm{~g} \cdot \mathrm{mol}^{-1}, 30 \mathrm{wt} \%$ in water), and Nafion (1100 E.W. (equivalent weight) perfluorinated resin solution, $5 \mathrm{wt} \%$ in lower aliphatic alcohols and $15-20 \%$ water) were purchased from SigmaAldrich. Sodium chloride was obtained from Akzo Nobel, The Netherlands, and sulfamethoxazole from Fluka. All other chemicals were obtained from Sigma-Aldrich. All chemicals were used without further purification.

Tight hollow fiber ultrafiltration membranes were obtained from NX Filtration B.V. (Enschede, The Netherlands). The membranes are asymmetric with the smallest pore size at the inner surface. The fibers have a positive surface charge, an inner diameter of $0.68 \mathrm{~mm}$, a standard permeability of $200 \mathrm{~L} \cdot \mathrm{m}^{-2} \cdot \mathrm{h}^{-1} \cdot \mathrm{bar}^{-1}$, and a molecular weight 
cutoff (MWCO) of $25 \mathrm{kDa}$. Silicon wafers were purchased from WaferNet Inc. (San Jose, CA, USA).

Dip Coating. Silicon wafers were first immersed, for $15 \mathrm{~min}$, in a solution of $0.1 \mathrm{~g} \cdot \mathrm{L}^{-1} \mathrm{PAH}$ and $0.5 \mathrm{M} \mathrm{NaCl}$ at a $\mathrm{pH}$ of 1.8 . Subsequently, they were rinsed three times for $5 \mathrm{~min}$ in separate baths containing $0.5 \mathrm{M} \mathrm{NaCl}$. After the rinsing steps, the wafers were immersed in a solution of $0.1 \mathrm{~g} \cdot \mathrm{L}^{-1}$ PSS and $0.5 \mathrm{M} \mathrm{NaCl}$. After the second dip-coating step, the silicon wafers were rinsed in the same manner as the first dip-coating step. This cycle completes one [PAH/ PSS] bilayer and is repeated with the same solutions until the desired number of [PAH/PSS ] bilayers is reached. For hollow fiber membranes the dip-coating procedure is similar, with all coating and rinsing solutions always containing the same concentration of $\mathrm{NaCl}(50,100,500$, or $1000 \mathrm{mM})$. However, for the hollow fiber membranes, the first step is to immerse them in the PSS solution instead of the PAH solution. Hollow fibers are completely immersed in an upright position in the coating and rinsing solutions to make sure that all air escapes from the inside of the fibers, such that the coating as well as the rinsing solutions are in contact with the entire inner surface of the fibers.

Nafion was coated on top of a $[\mathrm{PSS} / \mathrm{PAH}]_{\mathrm{n}}$ multilayer (PAH[PSS/ $\mathrm{PAH}]_{7}$ for silicon wafers) by dipping the hollow fiber membranes or silicon wafers in a solution of $80 / 20$ (wt \%) ethanol/water containing $0.1 \mathrm{~g} \cdot \mathrm{L}^{-1}$ of Nafion. The wafers and hollow fibers were first rinsed by a solution of $80 / 20$ (wt \%) ethanol/water and subsequently by demineralized water before storage in demineralized water.

When building a $[\mathrm{Nafion} / \mathrm{PAH}]$ multilayer, the demineralized water rinsing step is substituted by two rinsing steps containing the same ionic strength as the PAH coating solution. After rinsing, the fibers are immersed in a solution of $0.1 \mathrm{~g} \cdot \mathrm{L}^{-1} \mathrm{PAH}$, at a $\mathrm{pH}$ of 1.8 , and at the appropriate ionic strength. After PAH coating, the fibers were rinsed twice with the same ionic strength as the coating solution and one time in demineralized water; no $\mathrm{pH}$ adjustment to these rinsing solutions was performed. This cycle completes one [Nafion/PAH] bilayer; the cycle is repeated until the desired number of bilayers is obtained.

After dip coating is completed, the hollow fiber membranes are immersed in an aqueous solution containing $15 \mathrm{wt} \%$ glycerol for at least $4 \mathrm{~h}$. Subsequently, the hollow fiber membranes are dried overnight and potted into single hollow fiber modules with an approximate active fiber length of $17 \mathrm{~cm}$.

Ellipsometry. A rotating compensator ellipsometer (Mk-2000 V, J. A. Woollam Co., Inc.) was used for the spectroscopic ellipsometry measurements. The ellipsometer operates in a wavelength range of 370-1000 nm. First, wet thicknesses were measured for the as prepared wafers that were stored for at least $24 \mathrm{~h}$ in demineralized water. Wet thicknesses were measured by a $5 \mathrm{~mL}$ heated liquid cell filled with Milli-Q water at a $75^{\circ}$ angle of incidence under ambient temperature. The coated wafers were equilibrated for $30 \mathrm{~min}$ in the liquid cell before measuring. After measuring the wet thickness, the wafers were dried using a nitrogen gas flow. Dry thicknesses were measured under three different angles of incidence $\left(65^{\circ}, 70^{\circ}\right.$, and $75^{\circ}$ ) and at three different spots on the silicon wafers. All dry thickness measurements were done under ambient conditions.

$$
n(\lambda)=A+\frac{B}{\lambda^{2}}
$$

Data fitting was performed using the CompleteEase software. A Cauchy model, shown in eq 1 , was used to fit the data obtained from the coated silicon wafers. The refractive index of silica and its wavelength dependence are well-known and defined. For the polyelectrolyte multilayers, the Cauchy parameters were fitted independently from the thickness for layers thicker than $30 \mathrm{~nm}$. The swelling ratio for the multilayers was calculated using the dry and wet thickness, as shown by eq 2 . Average values and standard deviations of the thicknesses and swelling ratios were calculated and reported.

$$
\mathrm{SR}=\frac{d_{\mathrm{wet}}-d_{\mathrm{dry}}}{d_{\mathrm{dry}}} \times 100 \%
$$

Contact Angle. Contact angles were measured statically on a Dataphysics OCA15 plus instrument and calculated by using the SCA20 software. To determine the contact angle, a coated silicon wafer was placed on a flat surface. Subsequently, a $5 \mu \mathrm{L}$ water droplet (Milli-Q 18.2 M 2 ) was placed on the surface of the coated silicon wafer. To obtain the contact angle, a picture was taken $5 \mathrm{~s}$ after the water droplet touched the surface. All measurements were performed at least three times, and the average values and standard deviations were calculated and reported.

Membrane Resistance. The hydraulic resistance of the membranes toward water is determined by measuring the permeability of the polyelectrolyte multilayer membranes. At least three single hollow fiber modules were made for every membrane type and tested using a cross-flow setup operated by a rotary vane pump (BN71B4 pump motor, Bonfiglioli, Italy; IMTI $1.5 \mathrm{M}$ inverter, Electroil, Italy; PA411 pump head, Fluid-o-Tech, Italy). Permeability was measured at 7.9 bar of applied pressure under cross-flow conditions, and the resistance of the membranes was calculated using eq 3 below. In this equation, $\Delta P$ is the transmembrane pressure $(\mathrm{Pa})$, $\mu$ the dynamic viscosity $(\mathrm{Pa} \cdot \mathrm{s})$, and $J$ is the membrane flux $\left(\mathrm{m} \cdot \mathrm{s}^{-1}\right)$.

$$
R=\frac{\Delta P}{\mu J}
$$

Molecular Weight Cutoff. Molecular weight cutoff was determined with a feed mixture of several poly(ethylene glycol) (PEG) molecules of different molecular weights; PEG200, PEG400, PEG600, PEG1000, and PEG1500 at a concentration of $1 \mathrm{~g} \cdot \mathrm{L}^{-1}$ each. The cross-flow setup was operated at $1.7 \mathrm{bar}$ and a flow of $10 \mathrm{~L} \cdot \mathrm{h}^{-1}$ per module. Feed and permeate samples were analyzed via gel permeation chromatography with a size exclusion column (SEC, Agilent 1200/1260 Infinity GPC/SEC series, Polymer Standards Service data center and column compartment). Solutions were flown at $1 \mathrm{~mL} \cdot \mathrm{min}^{-1}$ over two Polymer Standards Service Suprema $8 \times 300$ $\mathrm{mm}$ columns in series: $1000 \AA, 10 \mu \mathrm{m}$ followed by $30 \AA, 10 \mu \mathrm{m}$. Concentrations were measured via refractive index measurements.

Salt Retention. Salt retention was measured in cross-flow mode using $5 \mathrm{mM}$ of $\mathrm{NaCl}, \mathrm{Na}_{2} \mathrm{SO}_{4}, \mathrm{MgCl}_{2}$, or $\mathrm{MgSO}_{4}$. The measurements were performed in the laminar flow regime at a cross-flow velocity of $1 \mathrm{~m} \cdot \mathrm{s}^{-1}$ (equal to a Reynolds number of 675) using a transmembrane pressure of 1.7 bar. To determine retention, the conductivity of both the permeate and feed was measured using a WTV 3210 conductivity meter. Using eq 4, the retention can be calculated on the basis of the difference in conductivity of the feed and permeate.

$$
R=\frac{\Delta C}{C_{\text {feed }}} \times 100 \%
$$

Micropollutant Retention. To measure micropollutant retentions, a cocktail was prepared containing $3 \mathrm{mg} \cdot \mathrm{L}^{-1}$ of each of the micropollutants (atenolol, atrazine, bezafibrate, bisphenol A, bromothymol blue, naproxen, phenolphthalein, and sulfamethoxazole) and adjusted to a $\mathrm{pH}$ of 5.8. The measurements were performed at equal flux; therefore, the cross-flow setup was operated at 1.2, 2.0, and 7,9 bar and at a cross-flow velocity of $1 \mathrm{~m} \cdot \mathrm{s}^{-1}$ per module. The separation process was allowed to stabilize for at least $24 \mathrm{~h}$ per measurement while leading permeate back into the feed, to prevent that adsorption leads to a higher apparent retention. Subsequently, around $2 \mathrm{~mL}$ of permeate was collected from each module and a calibration range was prepared from the feed. All samples were analyzed by HPLC (high-pressure liquid chromatography, Dionex Ultimate 3000; eluents, water, acetonitrile, and $0.1 \%$ phosphoric acid) over a Thermo Scientific Acclaim RSLC 120 C18 column $(2.2 \mu \mathrm{m}$, $2.1 \times 100 \mathrm{~mm}$ ). Micropollutant concentrations were determined via $\mathrm{UV} /$ vis detection at $225 \mathrm{~nm}$ and bromothymol blue at $430 \mathrm{~nm}$. Subsequently, retention was calculated in the same way as salt retention, using eq 4. 


\section{RESULTS AND DISCUSSION}

The results and discussion section is split into three distinct parts. In the first section, we focus on the characterization of $\mathrm{PSS} / \mathrm{PAH}$ and PSS/PAH/Nafion multilayers prepared on model surfaces (silicon wafers with a $1.8 \mathrm{~nm}$ top layer of silica). We utilize static contact angle and ellipsometry measurements to obtain a measure for the hydrophilicity, thickness, and swelling of the multilayer. Here, it is shown that the swelling and hydrophilic properties of the multilayer change when terminated with Nafion. In the second part, the same multilayer is built on porous hollow fiber support membranes and characterized by membrane permeability measurements in order to understand the effect of Nafion on the hydraulic resistance of the PEM membrane. In the third part, the modified membranes are assessed on their performance by salt retention, MWCO, and micropollutant retention measurements. By correlating the results of the model surface characterization with membrane performance, a much better understanding is achieved on how Nafion affects the properties and performance of PEM membranes.

Polyelectrolyte Multilayer Characterization: Model Surface. The effect of the terminating layer on a PSS/PAH multilayer was studied by contact angle measurements. From literature it is known that the contact angle of a PSS/PAH multilayer is around $30^{\circ}$ to $60^{\circ 29}$ and depends on the terminating layer. When PSS and PAH are alternately coated on a silicon wafer, a zigzag pattern is observed in the contact angle. It is known that the contact angle of a PAH terminated multilayer is $10^{\circ}$ to $25^{\circ}$ higher than when the multilayer is terminated with PSS. In this study, a PSS/PAH multilayer with 4.5 and 5.0 bilayers and 7.5 and 8.0 bilayers terminated either on PAH or PSS, respectively, give the expected contact angle of around $30^{\circ}$ to $60^{\circ}$, as shown in Table 1 . Nafion deposited as

Table 1. Contact angles of $[\mathrm{PAH} / \mathrm{PSS}]_{4} \mathrm{PAH}$ and $[\mathrm{PAH} /$ PSS $]_{7}$ PAH multilayers terminated by PAH, PSS, or Nafion. Both PAH and PSS were coated at $500 \mathrm{mM}$ of $\mathrm{NaCl}$ and the former at a $\mathrm{pH}$ of 1.8. Nafion was coated in a mixture of 80 / 20 wt.\% of ethanol/water. Error bars: standard deviation; $n$ $\geq 3$

\begin{tabular}{lclc} 
& $\begin{array}{c}\text { contact angle } \\
(\mathrm{deg})\end{array}$ & & $\begin{array}{c}\text { contact angle } \\
(\mathrm{deg})\end{array}$ \\
{$[\mathrm{PAH} / \mathrm{PSS}]_{4} \mathrm{PAH}$} & $43 \pm 1$ & {$[\mathrm{PAH} / \mathrm{PSS}]_{7} \mathrm{PAH}$} & $54 \pm 2$ \\
{$[\mathrm{PAH} / \mathrm{PSS}]_{5}$} & $38 \pm 1$ & {$[\mathrm{PAH} / \mathrm{PSS}]_{8}$} & $36 \pm 1$ \\
{$[\mathrm{PAH} / \mathrm{PSS}]_{4}$ Nafion } & $94 \pm 3$ & {$[\mathrm{PAH} / \mathrm{PSS}]_{8}$ Nafion } & $92 \pm 2$ \\
\hline
\end{tabular}

the final layer instead of PSS increases the contact angle of the total multilayer to around $95^{\circ}$. This obtained contact angle is in agreement with literature values of Nafion thin films; these films have a contact angle of $95^{\circ}$ to $106^{\circ} .{ }^{30,31}$ In addition, Ghoussoub and Schlenoff ${ }^{28}$ fabricated Nafion terminated PSS/ PDADMAC multilayers to make Janus nanofilms which have a hydrophobic and a hydrophilic side. The contact angles measured in their work of around $95^{\circ}$ are in agreement with contact angles obtained in this study. The results discussed here, clearly demonstrate that just a single layer of Nafion can significantly affect the PEM properties.

The same silicon wafers were studied by ellipsometry to determine the thickness both in dry and wet conditions. The dry thicknesses of the individual multilayers are $20 \pm 1,23 \pm$ 0.5 , and $23 \pm 1 \mathrm{~nm}$ for the 4.5 and 5.0 bilayer thick multilayer and $30 \pm 0.6,32 \pm 0.4$, and $33 \pm 0.3 \mathrm{~nm}$ for the 7.5 and 8.0 bilayer thick multilayers, terminated on PAH, PSS, and Nafion, respectively. From both the dry and wet thicknesses, the swelling ratio can be calculated according to eq 2; this result is plotted in Figure 2. Here, it can be observed that the swelling

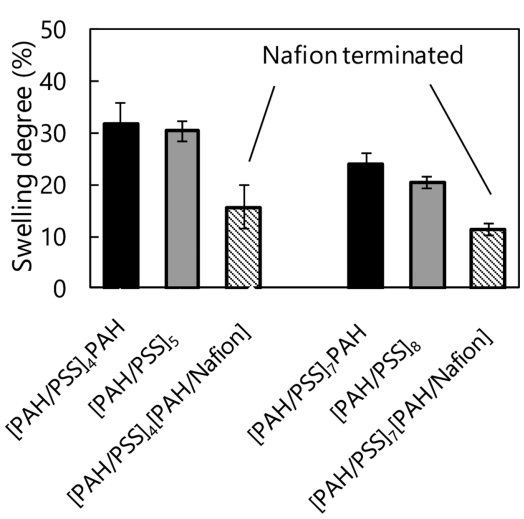

Figure 2. Swelling degree of a $[\mathrm{PAH} / \mathrm{PSS}]_{4} \mathrm{PAH}$ and $[\mathrm{PAH} /$ $\mathrm{PSS}]_{7} \mathrm{PAH}$ multilayer terminated by PAH, PSS, or Nafion. After measurement of the dry and wet thicknesses of the multilayers, eq 2 is used to calculate the swelling. Both PAH and PSS were coated at an ionic strength of $500 \mathrm{mM} \mathrm{NaCl}$, the $\mathrm{PAH}$ solution was adjusted to a $\mathrm{pH}$ of 1.8. Nafion was coated in a mixture of $80 / 20$ (wt \%) of ethanol/water. Error bars: standard deviation; $n \geq 3$.

of the 5.0 bilayer thick multilayer is always higher than the 8.0 bilayer thick multilayer with the same final layer. This observation is in agreement with literature where it is shown that a PSS/PAH multilayer is more hydrated at smaller layer numbers and that the swelling reaches a stable value of around $20 \%$ at higher layer numbers. ${ }^{29}$ Furthermore, it can be seen that the swelling degree changes significantly when the PSS/ $\mathrm{PAH}$ multilayer is terminated by a single layer of Nafion, with swelling degrees decreasing by half to $10 \%$ to $15 \%$. A Nafion terminated multilayer is thus much less hydrated than a normal PSS/PAH multilayer. For PEM-based membranes, it is wellestablished that the hydration of the layer is seen as a good indication of its separation performance, with a denser layer leading to higher hydraulic resistances for water permeation, but also to higher retention. ${ }^{11,32}$

Polyelectrolyte Multilayer Characterization: Hollow Fiber Membrane. In this part, the PSS/PAH multilayers were coated on porous hollow fiber supports by alternately dipping the supports in the corresponding polyelectrolyte solutions in the same manner as the silicon wafers. After a polyelectrolyte coating step, the hollow fiber membranes were rinsed in rinsing solutions with the same ionic strength as the polyelectrolyte dipping solutions. This procedure leads to similar coatings both on asymmetric hollow fibers supports and on the silicon wafers. ${ }^{11}$ When a PEM is coated on the support by means of dip coating, the inner as well as the outer support surface will be coated. However, a separation layer is formed only on the inside, because of the smaller pores that can be completely closed by the PEM. It is important to obtain $100 \%$ pore closure in order to have a defect-free separation layer. When two different polyelectrolytes are adsorbed alternately on the surface, a zigzag pattern in the permeability as a function of the number of layers is typically observed. ${ }^{11}$ This zigzag pattern stems from the so-called odd-even effect and results from the difference in multilayer swelling depending on the final layer. ${ }^{33}$ In the PEM membrane literature, the oddeven effect is used to distinguish the pore- and layer-dominated 

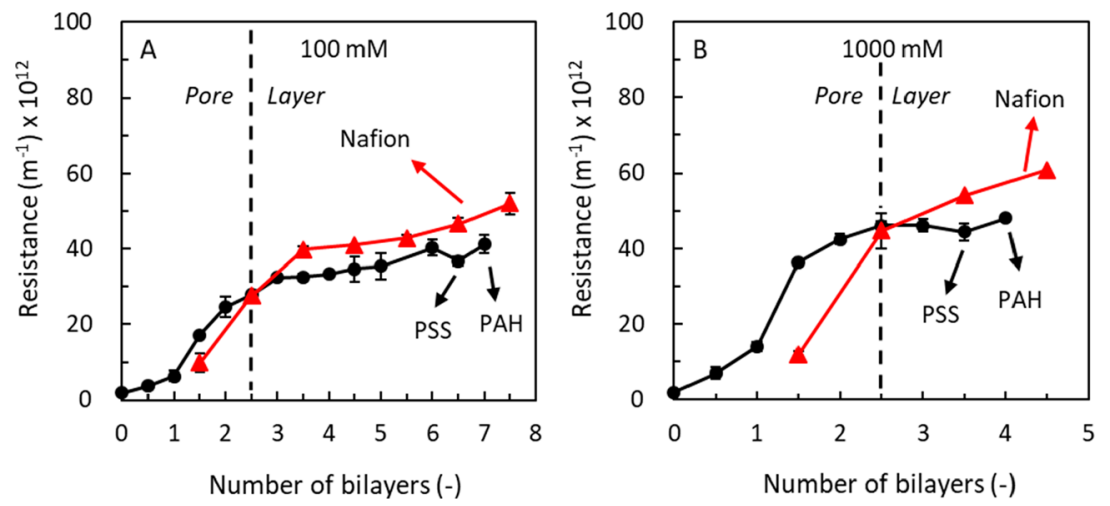

Figure 3. Hydraulic membrane resistance as a function of the number of bilayers for a PSS/PAH multilayer built at salt concentrations of 100 (A) and $1000 \mathrm{mM} \mathrm{NaCl}(\mathrm{B})$. The black line represents the $[\mathrm{PSS} / \mathrm{PAH}]_{n}$ multilayer at which PSS terminated multilayers are half-numbers and PAH terminated multilayers are full numbers. The red line represents a $[\mathrm{PSS} / \mathrm{PAH}]_{n} \mathrm{Nafion}$ multilayer that is terminated with one Nafion layer on top of a PAH layer. Error bars: standard deviation; $n \geq 3$.

regime. $^{11,15,34}$ During the initial coating steps, the PEM is coated on the inside of the pores and narrowing them, but the pores still define the separation properties until in the layerdominated regime, at which the pores are completely closed by the PEM. In the pore-dominated regime, the polyelectrolyte with the largest multilayer swelling has the highest hydraulic resistance against water permeation, as layer swelling directly leads to narrower pores. On the contrary, in the layerdominated regime the polyelectrolyte with the largest swelling has a lower hydraulic membrane resistance, as water can more easily permeate the more swollen layer. ${ }^{11}$ Therefore, changes in hydraulic resistance can provide important information on the structure of the PEM membrane.

In Figure 3a,b, the hydraulic membrane resistances of a $[\mathrm{PSS} / \mathrm{PAH}]_{n}$ multilayer (black line) and a $[\mathrm{PSS} / \mathrm{PAH}]_{n} \mathrm{Nafion}$ multilayer (red line) fabricated at 100 and $1000 \mathrm{mM} \mathrm{NaCl}$ are plotted against the number of bilayers. For both systems, a thicker multilayer leads to a higher hydraulic resistance, with an especially strong increase between 1 and 3 bilayers, indicative of the pores being closed by the PEM. For the PSS/PAH-based multilayer no strong zigzag effect is observed, in line with the small changes in hydration between PSS and PAH terminated layers (Figure 2). However, termination with Nafion leads to significantly different hydraulic membrane resistances, most likely due to the much lower hydration of the layer. Initially, for a multilayer of 1.5 bilayers thick, the hydraulic membrane resistance for Nafion terminated PEMs is lower than for the equally thick PSS terminated layers, in line with the effects expected for a pore-dominated regime where less swelling leads to a more open pore. For thicker layers (from 3.5 bilayers onward) we observe the opposite effect, the less hydrated Nafion terminated multilayers have a higher hydraulic membrane resistance than the PSS terminated layers. This is exactly what is expected in the layer-dominated regime, at which all water needs to permeate through the PEM and at which a less swollen PEM leads to a higher hydraulic resistance. At 2.5 bilayers, the hydraulic resistances for a Nafion and a PSS terminated multilayer are similar, which indicates a transition region between the pore- and layerdominated regime. Here, the low hydration of Nafion terminated PEMs provides very useful information on the structure of the prepared PEM membranes. It is observed that Nafion terminated multilayers lead directly to higher hydraulic membrane resistances, which could also be an indication for better selectivities. If we compare the data from the multilayers built at 100 and $1000 \mathrm{mM}$ of salt, the trends are similar. However, the increase in hydraulic resistance from Nafion termination seems to be higher at high salt concentrations, especially for thicker layers. This effect of ionic strength was subsequently studied in more detail.

In Figure 4, the hydraulic membrane resistance of PSS/PAH multilayers built at various salt concentrations $(50,100$, and

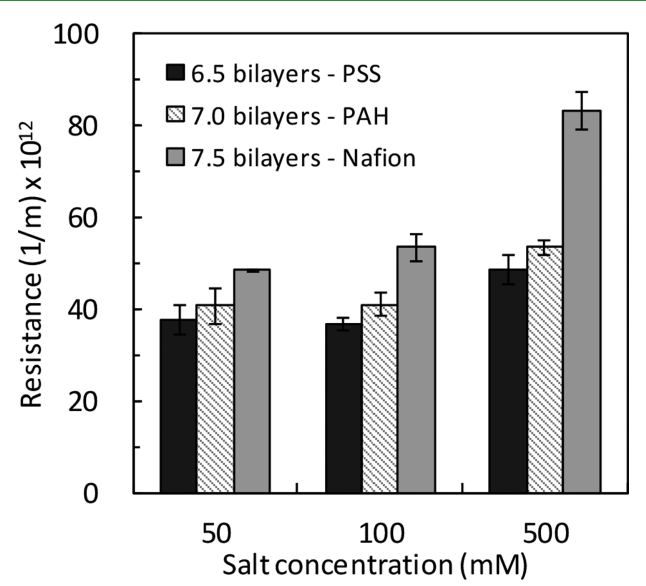

Figure 4. Multilayer in the layer-dominated regime at various salt concentrations. In both graphs the hydraulic resistance of the membrane as a function of the building salt concentration is plotted. Black bars represent a PSS terminated multilayer, patterned bars a $\mathrm{PAH}$ terminated multilayer, and gray bars a Nafion terminated multilayer. Error bars: standard deviation; $n \geq 3$.

$500 \mathrm{mM}$ ) is shown for layer thicknesses well in the layerdominated regime of $6.5,7$, and 7.5 bilayers thick. It can be observed at all salt concentrations, when terminating on PAH, that the hydraulic resistance increases compared with PSS terminated layers, since the multilayer thickness increases. Furthermore, the hydraulic resistance of the membrane increases when the multilayer is coated at higher salt concentrations. This increase in hydraulic resistance is due to the increased PEM thickness at higher ionic strength. When this multilayer is terminated by Nafion (gray bars), the increase in hydraulic resistance is much higher than at low ionic strength. This could be due to the increased mobility of polyelectrolyte chains, ${ }^{35}$ as Nafion chains could mix with the PSS/PAH layers below, which could lead to higher adsorption 
and a much stronger effect of Nafion as a final layer. At lower ionic strengths, a smaller effect on hydraulic resistance is observed, because less Nafion would be adsorbed due to the lowered mobility.

Membrane Performance. In the previous sections, polyelectrolyte multilayer (PEM) characterization was discussed, both on model surfaces and hollow fibers. We clearly demonstrated that by terminating a PSS/PAH multilayer with Nafion, the swelling decreases, resulting in an increased hydraulic membrane resistance. In this section, we study how these changes in layer structure affect the membrane separation properties by means of salt retention, MWCO, and micropollutant retention. We focus here on membranes prepared at high ionic strength, as in these conditions the change in hydraulic resistance was the most significant. Moreover, we compare layers terminated by either PSS or Nafion. Negatively charged membranes are highly favored for water treatment, ${ }^{36}$ and the comparison is more fair because the membrane surface charge can already significantly affect the retention of charged species. The retentions of various types of salts $\left(\mathrm{NaCl}, \mathrm{Na}_{2} \mathrm{SO}_{4}, \mathrm{MgCl}_{2}\right.$, and $\left.\mathrm{MgSO}_{4}\right)$ are measured to obtain a good understanding of the multilayer separation properties toward differently charged ions. Finally, we evaluate if the membranes could be made even denser by building a $[\mathrm{Nafion} / \mathrm{PAH}]_{6} \mathrm{Nafion}$ multilayer on top of the normal PSS/ PAH multilayer to increase the Nafion content. Permeabilities of the measured membranes for the salt retention and molecular weight cutoff are $6.2 \pm 0.4,4.8 \pm 0.7$, and $1.7 \pm$ $0.5 \mathrm{~L} \cdot \mathrm{m}^{-2} \cdot \mathrm{h}^{-1} \cdot \mathrm{bar}^{-1}$ for PSS, Nafion, and [Nafion/ $\mathrm{PAH}]_{6} \mathrm{Nafion}$ terminated $[\mathrm{PSS} / \mathrm{PAH}]_{8}$ multilayers, respectively. The salt retentions for $\mathrm{NaCl}, \mathrm{Na}_{2} \mathrm{SO}_{4}, \mathrm{MgCl}_{2}$, and $\mathrm{MgSO}_{4}$ are plotted in Figure 5 for the mentioned PEM membranes.

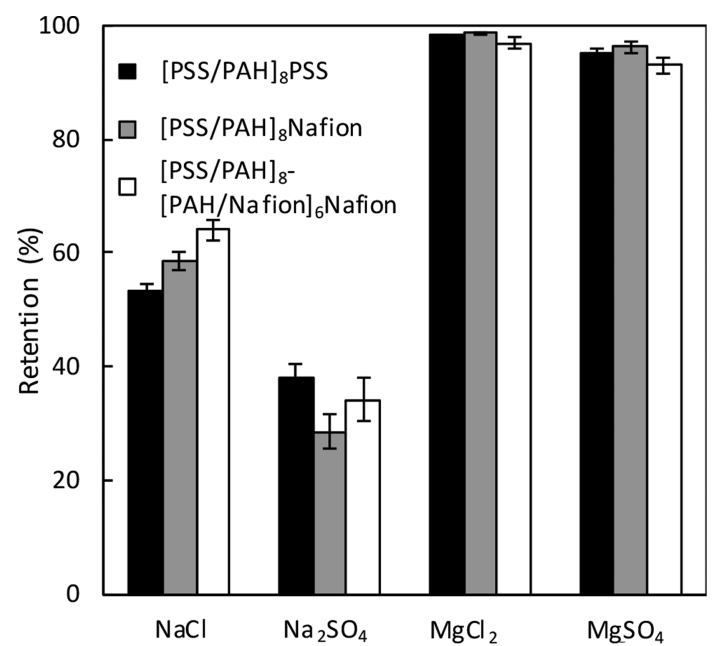

Figure 5. Salt retention toward $\mathrm{NaCl}, \mathrm{Na}_{2} \mathrm{SO}_{4}, \mathrm{MgCl}_{2}$, and $\mathrm{MgSO}_{4}$ for a $[\mathrm{PSS} / \mathrm{PAH}]_{8} \mathrm{PSS}$ (black bar), $[\mathrm{PSS} / \mathrm{PAH}]_{8} \mathrm{Nafion}$ (gray bar), and $[\mathrm{PSS} / \mathrm{PAH}]_{8}[\mathrm{Nafion} / \mathrm{PAH}]_{6}$ Nafion multilayer (white bar). Error bars: standard deviation; $n \geq 3$.

The retention was measured for the listed types of salts to study the retention properties of the membranes toward monovalent and divalent cations and anions. What can be observed in Figure 5 is that the PSS terminated multilayer shows a typical Donnan exclusion mechanism, with high retentions toward divalent cations $\left(\mathrm{MgCl}_{2}\right)$ and low retentions toward divalent anions $\left(\mathrm{Na}_{2} \mathrm{SO}_{4}\right){ }^{11}$ This could indicate that the bulk of the multilayer is positively charged and dominates ion retention. It is known for PSS/PAH multilayers that PAH is overcompensating in the multilayer ${ }^{37}$ and in addition, when the multilayer is PSS terminated, PAH is highly charged in the bulk of the multilayer. ${ }^{38,39}$ Retention toward $\mathrm{NaCl}$ slightly increases from $55 \%$ to $65 \%$, when the Nafion content is increased. Furthermore, retention toward $\mathrm{MgCl}_{2}$ and $\mathrm{MgSO}_{4}$ slightly changes but remain high with values around $98 \%$. On the other hand, retention toward $\mathrm{Na}_{2} \mathrm{SO}_{4}$ is low (around 38\%) for PSS terminated multilayer and decreases slightly when terminated with Nafion. Although Nafion influences the hydraulic resistance and hydration of the multilayer greatly, it is observed that the main separation principle for ions, the Donnan exclusion mechanism, is clearly not so much affected by the densification of terminating by Nafion.

A well-established method to determine how well membranes separate on size is to measure by means of MWCO experiments. With this method, poly(ethylene glycol) molecules with various molecular weights are filtered through the membrane and their retentions are determined by gel permeation chromatography (GPC). ${ }^{40,41}$ In this study, we use PEG molecules with molecular weights of $200,400,600$, 1000 , and $1500 \mathrm{~g} \cdot \mathrm{mol}^{-1}$. The results are shown in Table 2 and

Table 2. Molecular Weight Cutoff $\left(\mathrm{g} \cdot \mathrm{mol}^{-1}\right)$ of PEM Membranes with a $[\mathrm{PSS} / \mathrm{PAH}]_{8} \mathrm{PSS},[\mathrm{PSS} / \mathrm{PAH}]_{8}$ Nafion, and $[\mathbf{P S S} / \mathbf{P A H}]_{8}[\mathrm{Nafion} / \mathbf{P A H}]_{6}$ Nafion Multilayer As an Active Layer $^{a}$

$\begin{array}{lc} & \text { MWCO }\left(\mathrm{g} \cdot \mathrm{mol}^{-1}\right) \\ {[\mathrm{PSS} / \mathrm{PAH}]_{8} \mathrm{PSS}} & 301 \pm 5 \\ {[\mathrm{PSS} / \mathrm{PAH}]_{8} \text { Nafion }} & 287 \pm 8 \\ {[\mathrm{PSS} / \mathrm{PAH}]_{8}[\text { Nafion } / \mathrm{PAH}]_{6} \text { Nafion }} & 258 \pm 15\end{array}$

${ }^{a}$ MWCO was tested by permeating poly(ethylene glycol) with molecular weights of; 200, 400,600,1000, and $1500 \mathrm{~g} \cdot \mathrm{mol}^{-1}$ through the membrane. Error bars: standard deviation; $n \geq 3$.

the original sieving curves of the MWCO experiments are given in Figure S1 in the Supporting Information. From Table 2 , it can be seen that a PSS terminated multilayer has a MWCO of $301 \mathrm{~g} \cdot \mathrm{mol}^{-1}$; when the multilayer is Nafion terminated, the MWCO drops to $287 \mathrm{~g} \cdot \mathrm{mol}^{-1}$. Moreover, when a $[\mathrm{Nafion} / \mathrm{PAH}]_{6} \mathrm{Nafion}$ multilayer is constructed on top of the original PSS/PAH multilayer, the MWCO drops to a value of $258 \mathrm{~g} \cdot \mathrm{mol}^{-1}$. This indicates that an increasing amount of Nafion in the PEM creates a denser multilayer.

Subsequently, with the knowledge obtained in the previous sections about the multilayer characterization and the membrane performance measurements, we can move on toward our actual goal of retaining difficult-to-remove micropollutants. Furthermore, retaining these small micropollutants gives even more insight into the separation principles of our fabricated PEM membranes. For the micropollutant retention measurement, a cocktail is used of various small micropollutants, varying in charge and molecular weight, as shown in Figure 6. Many of these micropollutants are chosen on the basis of the most frequently encountered and urgent accumulating micropollutants in our water cycle. ${ }^{1}$ In addition, phenolphthalein and bromothymol blue are added to the cocktail to create a mix that covers a wide range of molecular weights and charges.

In order to compare each PEM membrane fairly, micropollutant retention is measured at equal permeate flux. In 
- $[\mathrm{PSS} / \mathrm{PAH}]_{8} \mathrm{PSS} \quad \square[\mathrm{PSS} / \mathrm{PAH}]_{8} \mathrm{Nafion} \quad \square[\mathrm{PSS} / \mathrm{PAH}]_{8}[\mathrm{Nafion} / \mathrm{PAH}]_{6} \mathrm{Nafion}$

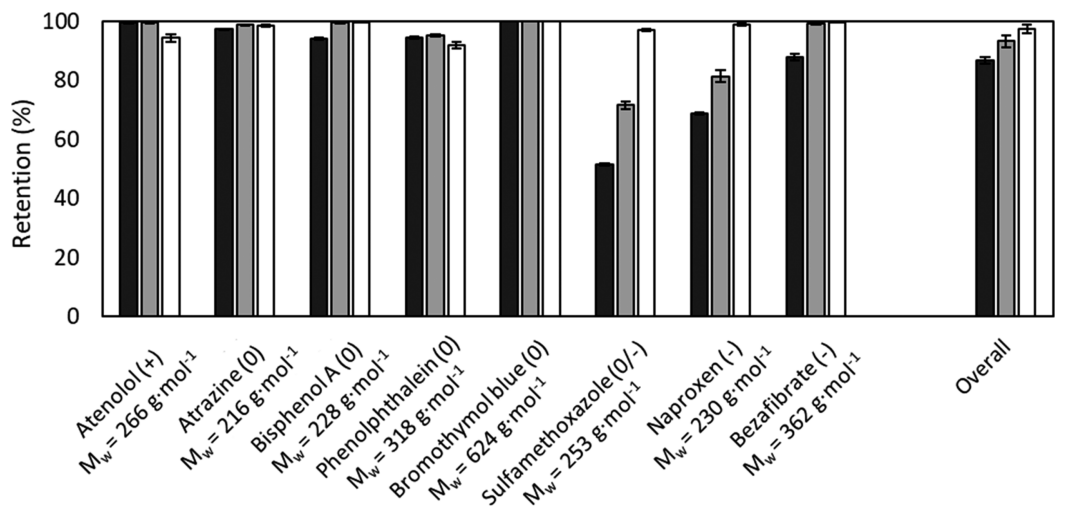

Figure 6. Micropollutant retention at equal flux $\left(5.5 \mathrm{~L} \cdot \mathrm{m}^{-2} \cdot \mathrm{h}^{-1}\right)$ for PSS (black column), Nafion (gray column), and $[\mathrm{Nafion} / \mathrm{PAH}]_{6} \mathrm{Nafion}($ white column) terminated $[\mathrm{PSS} / \mathrm{PAH}]_{8} \mathrm{PEM}$ membranes. Micropollutants are sorted by charge, positive to negative from left to right. Retention was measured of a cocktail of micropollutants with a concentration of $3 \mathrm{mg} \cdot \mathrm{L}^{-1}$ per micropollutant at a $\mathrm{pH}$ of 5.8 . Error bars: standard error; $n=5$.

addition, to ensure that adsorption is not included in the measured membrane retention, tests were performed after filtering the micropollutant solution for at least $24 \mathrm{~h}^{42}$ Subsequently, the permeate is collected and further analyzed by HPLC to detect any micropollutants in the permeate; these results are plotted in Figure 6. Here, it can be observed that all multilayers show a complete removal of bromothymol blue $\left(624 \mathrm{~g} \cdot \mathrm{mol}^{-1}\right)$; this clearly shows that our membranes are defect-free. The PSS terminated multilayer shows a high retention toward the positively charged atenolol, most likely due to excess $\mathrm{PAH}$ present inside the PSS/PAH multilayer as described earlier. For this reason, positively charged species are rejected from the bulk of the membrane even though the multilayer is terminated by the negatively charged PSS. Overall, the $[\mathrm{PSS} / \mathrm{PAH}]_{8} \mathrm{PSS}$ multilayer performs already very well in retaining these small micropollutants when compared to commercial alternatives. ${ }^{43,44}$ This multilayer already has an overall retention of $87 \%$ and retentions above 90\% for atenolol, atrazine, bisphenol A, and phenolphthalein. However, retention toward bezafibrate, naproxen, and especially SMX can still be greatly improved by making the multilayer denser.

Terminating the multilayer by a single layer of Nafion results in an increased retention toward atrazine (97\% to 99\%), bisphenol A (94\% to $99.5 \%$ ), sulfamethoxazole (SMX, 52\% to $72 \%$ ), naproxen (69\% to $81 \%$ ), and bezafibrate ( $88 \%$ to $99 \%$ ) for a $[\mathrm{PSS} / \mathrm{PAH}]_{8} \mathrm{Nafion}$ in comparison with a $[\mathrm{PSS} /$ $\mathrm{PAH}]_{8}$ PSS multilayer at equal permeate flux. Overall, the retention for the tested micropollutants increases from $87 \%$ to $93 \%$ by adding just a single layer of Nafion. An increase in overall retention from $87 \%$ to $93 \%$ might seem small, but it means that micropollutant permeation through the membrane is reduced by a factor 2 . Thus, the effective selectivity of the membrane is doubled as these retentions are obtained at equal flux. This is in line with the ellipsometry results discussed earlier where we showed that the multilayer becomes denser.

When a $[\mathrm{Nafion} / \mathrm{PAH}]_{6} \mathrm{Nafion}$ multilayer is built on top of the $[\mathrm{PSS} / \mathrm{PAH}]_{8}$ multilayer, thereby increasing the Nafion content of the multilayer, micropollutant retentions increase overall from $93 \%$ to $97 \%$ in comparison to a [PSS/ $\mathrm{PAH}]_{8} \mathrm{Nafion}$ multilayer, doubling membrane selectivity again. When the $[\mathrm{Nafion} / \mathrm{PAH}]_{6}$ Nafion multilayer is compared to the $[\mathrm{PSS} / \mathrm{PAH}]_{8} \mathrm{PSS}$, overall retention increases from $87 \%$ to $97 \%$. This means that $77 \%$ less micropollutants permeate the membrane and that the effective selectivity for water to MPs is quadrupled as a result of this high Nafion content. Especially, high increases in retentions are observed for SMX and naproxen; however, it is noticed that the retention toward atenolol and phenolphthalein decreases slightly. Overall, it can be observed that an increasing Nafion content within the multilayer ensures that small and difficult-to-remove micropollutants are retained to a much higher degree. This is completely in line with the layer densification observed on model surfaces (Figure 2) and membranes (Figure 3).

In comparison to commercial NF and reverse osmosis (RO) membranes, the PSS terminated membranes fabricated in this study have permeabilities comparable to commercial NF membranes, whereas the Nafion terminated membranes have permeabilities in the $\mathrm{RO}$ regime. Literature reports retentions toward bisphenol $\mathrm{A}$ in the range of $11 \%$ to $99 \%$ and SMX retentions of $70 \%$ to $96 \%$ depending on which type of RO membrane is used. ${ }^{43-45}$ The PSS/PAH/Nafion membranes produced in this study have retentions higher than $99 \%$ toward bisphenol A and between $72 \%$ to $97 \%$ toward SMX depending on the amount of Nafion used in the PEM membranes. These retention values are consistently higher than the retentions reported in literature. Moreover, we reach these retentions on the basis of layer-by-layer assembly, which has considerable advantages such as easy application on the inner surface of hollow fiber membranes and easy upscaling, although the technique is laborious like many other dip-coating techniques. We have shown that a PSS/PAH membrane formed via this method has already high micropollutant retentions and that we can improve these properties by densifying the layer with Nafion.

\section{CONCLUSION}

In this study, we show that by simply terminating a PSS/PAH polyelectrolyte multilayer (PEM) with Nafion, properties of the PEM membrane can be changed. By using Nafion, a polymer commonly used for making ion exchange membranes, the selectivity toward small and difficult-to-remove micropollutants can be greatly enhanced. On silicon wafers, which act as model surfaces, we show that the swelling of a PSS/PAH multilayer decreases by $50 \%$ when it is terminated by Nafion. Furthermore, the contact angle of the multilayer changes from $30^{\circ}$ to $60^{\circ}$ to around $95^{\circ}$. This means that the hydrophobic character of Nafion determines the properties of the multilayer, 
making it less hydrated and more hydrophobic. This change in multilayer properties can be directly connected to the performance of the membrane.

The MWCO goes down from 301 to $258 \mathrm{~g} \cdot \mathrm{mol}^{-1}$ when the Nafion content of the PSS/PAH/Nafion multilayer increases. Furthermore, the hydraulic resistance of the membrane toward water is doubled when the multilayer is terminated by a single layer of Nafion and increases even more when the Nafion content is increased. This is in line with the observed decrease in swelling, and in combination with the lowering MWCO, this directly translates in an overall retention toward small and difficult-to-remove micropollutants $(200-650 \mathrm{Da})$ that increases from $87 \%$ to $97 \%$. This means that the selectivity of the membrane is quadrupled, while the same salt retentions are maintained. A sharp increase in retention was observed especially for bisphenol A, sulfamethoxazole, naproxen, and bezafibrate when the Nafion content was introduced and increased. Compared with micropollutant retentions as reported in literature, the membranes produced in this study show higher retention values.

This work shows that PEM membranes can be optimized toward a high micropollutant retention, in which low hydration of the multilayer plays an important role. This knowledge can be used to further optimize PEM membranes for micropollutant removal.

\section{ASSOCIATED CONTENT}

\section{S Supporting Information}

The Supporting Information is available free of charge on the ACS Publications website at DOI: 10.1021/acsapm.9b00689.

Molecular weight cutoff sieving curves (PDF)

\section{AUTHOR INFORMATION}

\section{Corresponding Author}

*E-mail: w.m.devos@utwente.nl.

\section{ORCID $\odot$}

Wiebe M. de Vos: 0000-0002-0133-1931

\section{Notes}

The authors declare no competing financial interest.

\section{ACKNOWLEDGMENTS}

This project was made possible through financial support of Aquaporin A/S (Lyngby, Denmark), and the TKI HTSM, through the University of Twente Impuls program. This project has received funding from the European Research Council (ERC) under the European Union's Horizon 2020 research and innovation programme (Grant ERC StG 714744 SAMBA).

\section{REFERENCES}

(1) de Voogt, P.; Janex-Habibi, M. L.; Sacher, F.; Puijker, L.; Mons, M. Development of a common priority list of pharmaceuticals relevant for the water cycle. Water Sci. Technol. 2009, 59 (1), 39-46.

(2) Tröger, R.; Klöckner, P.; Ahrens, L.; Wiberg, K. Micropollutants in drinking water from source to tap - Method development and application of a multiresidue screening method. Sci. Total Environ. 2018, 627, 1404-1432.

(3) Geissen, V.; Mol, H.; Klumpp, E.; Umlauf, G.; Nadal, M.; van der Ploeg, M.; van de Zee, S. E. A. T. M.; Ritsema, C. J. Emerging pollutants in the environment: A challenge for water resource management. Int. Soil Water Conserv. Res. 2015, 3, 57-65.
(4) Stuart, M.; Lapworth, D.; Crane, E.; Hart, A. Review of risk from potential emerging contaminants in UK groundwater. Sci. Total Environ. 2012, 416, 1-21.

(5) Baker, R. W., Overview of membrane science and technology. Membrane technology and applications, 2 ed.; John Wiley \& Sons: West Sussex, England, 2004; pp 1-14, DOI: 10.1002/0470020393.ch1.

(6) Yaroshchuk, A. E. Dielectric exclusion of ions from membranes. Adv. Colloid Interface Sci. 2000, 85 (2-3), 193-230.

(7) Szymczyk, A.; Fievet, P. Ion transport through nanofiltration membranes: the steric, electric and dielectric exclusion model. Desalination 2006, 200, 122-124.

(8) Lau, W. J.; Ismail, A. F.; Misdan, N.; Kassim, M. A. A recent progress in thin film composite membrane: A review. Desalination 2012, 287, 190-199.

(9) de Grooth, J.; Haakmeester, B.; Wever, C.; Potreck, J.; de Vos, W. M.; Nijmeijer, K. Long term physical and chemical stability of polyelectrolyte multilayer membranes. J. Membr. Sci. 2015, 489, 153159.

(10) Krasemann, L.; Tieke, B. Selective ion transport across selfassembled alternating multilayers of cationic and anionic polyelectrolytes. Langmuir 2000, 16 (2), 287-290.

(11) de Grooth, J.; Oborný, R.; Potreck, J.; Nijmeijer, K.; de Vos, W. $M$. The role of ionic strength and odd-even effects on the properties of polyelectrolyte multilayer nanofiltration membranes. J. Membr. Sci. 2015, 475, 311-319.

(12) Shiratori, S. S.; Rubner, M. F. pH-dependent thickness behavior of sequentially adsorbed layers of weak polyelectrolytes. Macromolecules 2000, 33 (11), 4213-4219.

(13) Ilyas, S.; English, R.; Aimar, P.; Lahitte, J.-F.; de Vos, W. M. Preparation of multifunctional hollow fiber nanofiltration membranes by dynamic assembly of weak polyelectrolyte multilayers. Colloids Surf., A 2017, 533, 286-295.

(14) Jin, W.; Toutianoush, A.; Tieke, B. Use of Polyelectrolyte Layer-by-Layer Assemblies as Nanofiltration and Reverse Osmosis Membranes. Langmuir 2003, 19 (7), 2550-2553.

(15) Reurink, D. M.; Haven, J. P.; Achterhuis, I.; Lindhoud, S.; Roesink, H. D. W.; de Vos, W. M. Annealing of Polyelectrolyte Multilayers for Control over Ion Permeation. Adv. Mater. Interfaces 2018, 5 (20), 1800651.

(16) Cheng, C.; Yaroshchuk, A.; Bruening, M. L. Fundamentals of selective ion transport through multilayer polyelectrolyte membranes. Langmuir 2013, 29 (6), 1885-1892.

(17) Adusumilli, M.; Bruening, M. L. Variation of ion-exchange capacity, zeta potential, and ion-transport selectivities with the number of layers in a multilayer polyelectrolyte film. Langmuir 2009, 25 (13), 7478-7485.

(18) Jin, W.; Toutianoush, A.; Tieke, B. Size- and charge-selective transport of aromatic compounds across polyelectrolyte multilayer membranes. Appl. Surf. Sci. 2005, 246, 444-450.

(19) Liu, X.; Bruening, M. L. Size-Selective Transport of Uncharged Solutes through Multilayer Polyelectrolyte Membranes. Chem. Mater. 2004, 16 (2), 351-357.

(20) Xu, T. W. Ion exchange membranes: State of their development and perspective. J. Membr. Sci. 2005, 263 (1-2), 1-29.

(21) Luo, T.; Abdu, S.; Wessling, M. Selectivity of ion exchange membranes: A review. J. Membr. Sci. 2018, 555, 429-454.

(22) Abdu, S.; Marti-Calatayud, M. C.; Wong, J. E.; GarciaGabaldon, M.; Wessling, M. Layer-by-layer modification of cation exchange membranes controls ion selectivity and water splitting. ACS Appl. Mater. Interfaces 2014, 6 (3), 1843-1854.

(23) White, N.; Misovich, M.; Alemayehu, E.; Yaroshchuk, A.; Bruening, M. L. Highly selective separations of multivalent and monovalent cations in electrodialysis through Nafion membranes coated with polyelectrolyte multilayers. Polymer 2016, 103, 478-485.

(24) Daiko, Y.; Sakamoto, H.; Katagiri, K.; Muto, H.; Sakai, M.; Matsuda, A. Deposition of Ultrathin Nafion Layers on Sol-GelDerived Phenylsilsesquioxane Particles via Layer-by-Layer Assembly. J. Electrochem. Soc. 2008, 155 (5), B479-B482. 
(25) Daiko, Y.; Katagiri, K.; Matsuda, A. Proton Conduction in Thickness-Controlled Ultrathin Polycation/Nafion Multilayers Prepared via Layer-by-Layer Assembly. Chem. Mater. 2008, 20 (20), 6405-6409.

(26) Daiko, Y.; Katagiri, K.; Yazawa, T.; Matsuda, A. Thickness dependences of proton conductivity for ultrathin Nafion multilayers prepared via layer-by-layer assembly. Solid State Ionics 2010, 181 (34), 197-200.

(27) Abebe, D. G.; Farhat, T. R. Self-assembly of Nafion ${ }^{\circledR} /$ poly(vinyl alcohol) at $\mathrm{pH}=1.2$ and $\mathrm{Nafion}{ }^{\circledR} /$ poly(allyl amine) at $\mathrm{pH}$ = 11. Soft Matter 2010, 6, 1325-1335.

(28) Ghoussoub, Y. E.; Schlenoff, J. B. Janus Nanofilms. Langmuir 2016, 32 (15), 3623-9.

(29) Wong, J. E.; Rehfeldt, F.; Hänni, P.; Tanaka, M.; Klitzing, R. v. Swelling Behavior of Polyelectrolyte Multilayers in Saturated Water Vapor. Macromolecules 2004, 37 (19), 7285-7289.

(30) Paul, D. K.; Karan, K.; Docoslis, A.; Giorgi, J. B.; Pearce, J. Characteristics of Self-Assembled Ultrathin Nafion Films. Macromolecules 2013, 46 (9), 3461-3475.

(31) Paul, D.; Fraser, A.; Pearce, J.; Karan, K. Understanding the Ionomer Structure and the Proton Conduction Mechanism in PEFC Catalyst Layer: Adsorbed Nafion on Model Substrate. ECS Trans. 2011, 41 (1), 1393-1406.

(32) Rijnaarts, T.; Reurink, D. M.; Radmanesh, F.; de Vos, W. M.; Nijmeijer, K. Layer-by-layer coatings on ion exchange membranes: Effect of multilayer charge and hydration on monovalent ion selectivities. J. Membr. Sci. 2019, 570-571, 513-521.

(33) Schönhoff, M.; Ball, V.; Bausch, A. R.; Dejugnat, C.; Delorme, N.; Glinel, K.; Klitzing, R. V.; Steitz, R. Hydration and internal properties of polyelectrolyte multilayers. Colloids Surf., A 2007, 303 (1-2), 14-29.

(34) Ilyas, S.; de Grooth, J.; Nijmeijer, K.; de Vos, W. M. Multifunctional polyelectrolyte multilayers as nanofiltration membranes and as sacrificial layers for easy membrane cleaning. J. Colloid Interface Sci. 2015, 446, 386-393.

(35) Schlenoff, J. B.; Dubas, S. T. Mechanism of Polyelectrolyte Multilayer Growth: Charge Overcompensation and Distribution. Macromolecules 2001, 34 (3), 592-598.

(36) Kumar, R.; Ismail, A. F. Fouling control on microfiltration/ ultrafiltration membranes: Effects of morphology, hydrophilicity, and charge. J. Appl. Polym. Sci. 2015, 132 (21), 42042.

(37) Riegler, H.; Essler, F. Polyelectrolytes. 2. Intrinsic or extrinsic charge compensation? Quantitative charge analysis of PAH/PSS multilayers. Langmuir 2002, 18 (17), 6694-6698.

(38) de Vos, W. M.; Mears, L. L. E.; Richardson, R. M.; Cosgrove, T.; Barker, R.; Prescott, S. W. Nonuniform Hydration and Odd-Even Effects in Polyelectrolyte Multilayers under a Confining Pressure. Macromolecules 2013, 46 (3), 1027-1034.

(39) Abbott, S. B.; de Vos, W. M.; Mears, L. L. E.; Barker, R.; Richardson, R. M.; Prescott, S. W. Hydration of Odd-Even Terminated Polyelectrolyte Multilayers under Mechanical Confinement. Macromolecules 2014, 47 (10), 3263-3273.

(40) Rohani, R.; Hyland, M.; Patterson, D. A refined one-filtration method for aqueous based nanofiltration and ultrafiltration membrane molecular weight cut-off determination using polyethylene glycols. $J$. Membr. Sci. 2011, 382 (1-2), 278-290.

(41) Xu, L. L.; Shahid, S.; Shen, J. J.; Emanuelsson, E. A. C.; Patterson, D. A. A wide range and high resolution one-filtration molecular weight cut-off method for aqueous based nanofiltration and ultrafiltration membranes. J. Membr. Sci. 2017, 525, 304-311.

(42) Abtahi, S. M.; Marbelia, L.; Gebreyohannes, A. Y.; Ahmadiannamini, P.; Joannis-Cassan, C.; Albasi, C.; de Vos, W. M.; Vankelecom, I. F. J. Micropollutant rejection of annealed polyelectrolyte multilayer based nanofiltration membranes for treatment of conventionally-treated municipal wastewater. Sep. Purif. Technol. 2019, 209, 470-481.

(43) Kimura, K.; Toshima, S.; Amy, G.; Watanabe, Y. Rejection of neutral endocrine disrupting compounds (EDCs) and pharmaceutical active compounds (PhACs) by RO membranes. J. Membr. Sci. 2004, 245 (1-2), 71-78.

(44) Comerton, A. M.; Andrews, R. C.; Bagley, D. M.; Hao, C. Y. The rejection of endocrine disrupting and pharmaceutically active compounds by NF and RO membranes as a function of compound a water matrix properties. J. Membr. Sci. 2008, 313 (1-2), 323-335.

(45) Yuksel, S.; Kabay, N.; Yuksel, M. Removal of bisphenol A (BPA) from water by various nanofiltration (NF) and reverse osmosis (RO) membranes. J. Hazard. Mater. 2013, 263 (Pt 2), 307-310. 